\title{
RF and mm-Wave SOP Module Platform using LCP and RF MEMS Technologies
}

\author{
S.Sarkar, V.Palazarri, G.Wang, N. Papageorgiou, D. Thompson, J.H.Lee, \\ S.Pinel, M.M.Tentzeris, J.Papapolymerou, J.Laskar. \\ Georgia Electronic Design Center, School of Electrical and Computer Engineering \\ Georgia Institute of Technology, Atlanta GA 30332-0269 USA \\ Fax:(404) 894-0222, Email; saikat @ece.gatech.edu
}

\begin{abstract}
In this paper, we present a liquid crystal polymer (LCP) based multilayer packaging technology combined with RF-MEMS technology and its emergence as an ideal platform for low cost, multi-band and reconfigurable $\mathrm{RF}$ front-end module integration. LCP's very low water absorption $(0.04 \%)$, low cost and high electrical performance makes it very appealing for RF applications. Here we describe the fabrication process of LCP substrate, its characterization and properties upto $W$ band, design of high $Q$ inductors, SISO dual band filters with insertion loss as low as $2.4 \mathrm{~dB}$ in $\mathrm{L}$ band and $1.8 \mathrm{~dB}$ in $\mathrm{C}$ band respectively. MEMS-SOP switch fabrication and finally integration of $C$ band wireless LAN (WLAN) module demonstrates the potential for compact, multiband and reconfigurable systems. This is the first complete report on the combination of LCP with RF-MEMS technology as a new approach towards the system-on-package (SOP) solutions for wireless communication applications.
\end{abstract}

Index Terms - LCP, reconfigurable and multiband module, dual band filter, MEMS switch on LCP, WLAN module, SOP, multilayer RF technologies.

\section{INTRODUCTION}

Miniaturization, portability, cost and performance have been the driving force for the evolution of packaging and system-on-package (SOP) approach in RF, microwave and millimeter wave applications. Recent research shows SOP to be a more feasible and low cost solution than systemon-chip (SOC) approach [1]. Liquid crystal polymer (LCP) provides the all-in-one solution for such integration approach in terms of high quality dielectric for high performance multiband passive design, excellent substrate for heterogeneous SOP integration as well as for MEMS structures. Furthermore low loss and flexible MEMS switches fabricated on LCP enable the implementation of multiband and reconfigurable modules.

In this paper, we present the potential of LCP as the substrate as well as the packaging material for wireless applications. The section II of this paper discusses the LCP fabrication process, its characterization and the design of high Q ( 90) integrated inductor s in L band. Section III analyzes the novel design of SISO dual band filters for $\mathrm{L}$ and $\mathrm{C}$ band with $2.4 \mathrm{~dB}$ and $1.8 \mathrm{~dB}$ of insertion loss respectively. Section IV concentrates on the fabrication of MEMS switches on LCP substrate with insertion loss of $0.1 \mathrm{~dB}$ up to $20 \mathrm{GHz}$. The implementation of IEEE802.11a compliant WLAN module on LCP is presented in section $\mathrm{V}$. The receiver shows a high sensitivity $(\sim-70 \mathrm{dBm})$, low noise figure $(<8 \mathrm{~dB})$ and high $\mathrm{LO}$ leakage suppression of $55 \mathrm{~dB}$. The transmitter works at a $6 \mathrm{~dB}$ back off from output $\mathrm{P}_{1} \mathrm{~dB}$ of $30 \mathrm{dBm}$. Hence we present the first complete report on LCP integrated RF, mm-wave functions and modules.

\section{LCP PROCESS AND INTEGRATION CONCEPT}

LCP is a fairly new, low cost thermoplastic material and its unique performance for an organic material is comparable to ceramic-based substrates that are widely used in RF and microwave applications. Its dielectric constant is 2.9 at $5.8 \mathrm{GHz}$ and increases very slightly with frequency up to $110 \mathrm{GHz}$, while the loss tangent is very small $(\sim 0.002)$. The low coefficient of thermal expansion (CTE) $\left(8-17 * 10^{-6}\right)$ leads to better matching to silicon or chip package and provides better reliability. The low moisture absorption $(\sim 0.04 \%)$ enables a better stability of performances. LCP offers large area processing capability that leads to tremendous cost reduction compared to commonly used LTCC substrate.

The loss characterization of LCP transmission lines up to $\mathrm{W}$ band provides an excellent insight of its potential for mm-wave applications. Conductor backed CPW (CBCPW) transmission lines have been fabricated on $2 \mathrm{mil}$ thick LCP substrates with measured insertion loss of $2.24 \mathrm{~dB} / \mathrm{cm}$ at $110 \mathrm{GHz}$ [2].

LCP has also been proven to be an excellent material to design high $\mathrm{Q}$ spiral inductors. The measured results exhibit very good quality factors as high as 90 from $\mathrm{C}$ to $\mathrm{X}$-band, for inductance values ranging from 2 to $5 \mathrm{nH}$ [3].

The low cost, low loss and easy integrability of LCP has already been addressed in [1]. But the fabrication of SISO dual band filters and MEMS switches extend the platform to multiband and reconfigurable applications. 
Figure 1 illustrates the proposed module concept. It consists of a LCP multilayer substrate including embedded high performance passives, such as dual band filter, dual band antenna and RF-MEMS switch directly fabricated on the LCP substrate, as integration platform for reconfigurable and multiband RF microwave module.

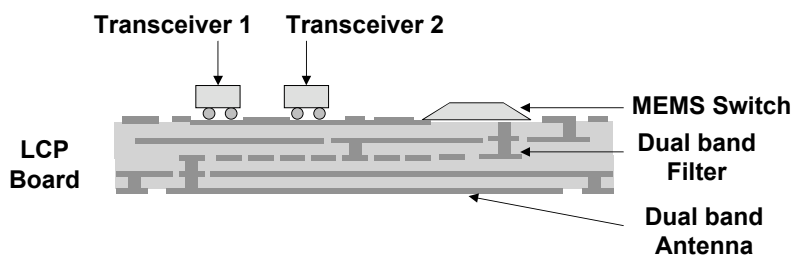

Fig 1. Multiband and reconfigurable module integration concept on LCP

\section{DUAL BAND FILTER}

Single-Input-Single-Output (SISO) LCP dual-band filter has been synthesized based on the novel "dual behaviour resonator" technique and has been successfully fabricated on LCP as shown in Fig 2. The purpose of this filter is to provide the same RF path to multi-standard and multi-band signals. It does not require parallel combination of two filters to achieve the multiband nature. The WLAN operating frequency bands, ISM $2.4 \mathrm{GHz}$ and UNII $5 \mathrm{GHz}$, have been targeted because of the ever-growing number of services allocated in this part of the spectrum, including Bluetooth, IEEE $802.11 \mathrm{a} / \mathrm{b} / \mathrm{g}$, and the introduction of dualband wireless systems.

The dual behaviour resonators (DBRs) have been realized by associating two open-ended stubs [4]. The open stubs cause insertion of transmission nulls, whose resonance frequency can be easily controlled by adjusting the stub length. To achieve bandpass response a third resonator has been added to create a third transmission null in order to obtain a dual band narrow band pass filter. On this basis, the stub dimensions have been optimized in order to have transmission nulls at $2.2 \mathrm{GHz}, 2.93 \mathrm{GHz}$ and $3.14 \mathrm{GHz}$.

It is worth noting that the desired bands, $2.4-2.5 \mathrm{GHz}$ and 5.15-5.85 GHz, are very different in terms of width (4\% bandwidth at $2.4 \mathrm{GHz}, 14 \%$ bandwidth at $5 \mathrm{GHz}$ ). Moreover the channel spacing is wide and a good rejection is difficult to achieve with the standard technique. To realize the pass-band in the $5 \mathrm{GHz}$ range, the second resonance frequency of the first stub has been successfully exploited (the first and the second harmonic being at 4.4 and $6.6 \mathrm{GHz}$ ), while the close transmission nulls at 2.9 and $3.14 \mathrm{GHz}$ allow for a better rejection in the inner stop band. To achieve better selectivity a second order filter has been implemented. They have been properly connected under constructive recombination criteria (using a $\square / 4$ line).

Fig. 3 shows very good agreement between simulations and measurements. Process variations explain the frequency shift (mainly substrate thickness) and the parasitic behavior at $2.9 \mathrm{GHz}$ (variation in the positioning of the transmission nulls at $2.93 \mathrm{GHz}$ and $3.14 \mathrm{GHz}$ ). The insertion loss at the central frequency is $2.4 \mathrm{~dB}$ for the $2.4 \mathrm{GHz}$ band and $1.8 \mathrm{~dB}$ for the $5 \mathrm{GHz}$ band. Return losses are $15 \mathrm{~dB}$ and $10 \mathrm{~dB}$ respectively. Out of band rejection is greater than $45 \mathrm{~dB}$ in between $\mathrm{L}$ and $\mathrm{C}$ band at $3.14 \mathrm{GHz}$.

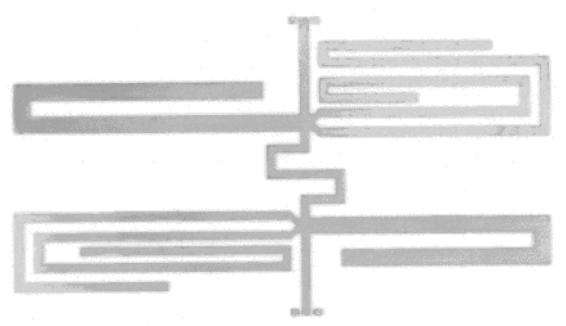

Fig 2. Photo of fabricated SISO dual band filter on LCP

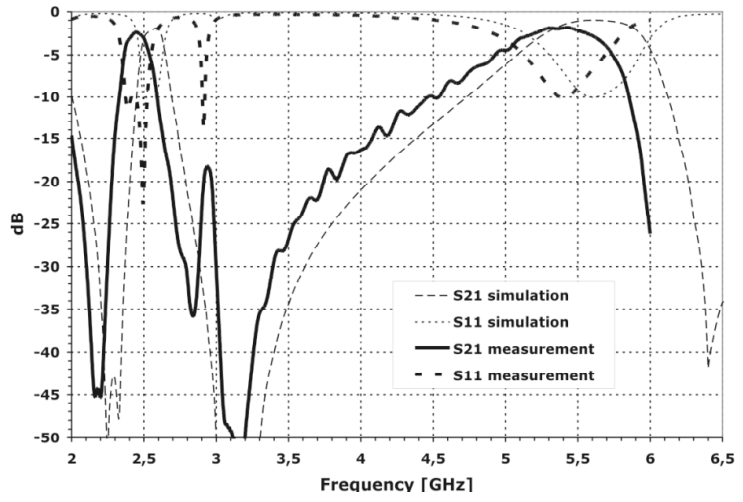

Fig 3. Performance of the SISO dual band filter on LCP

\section{MEMS SWITCH ON LCP}

Various designs of capacitive RF micromechanical switches made out of nickel [5], aluminum, gold and copper have been so far reported in literature, with a variety of applications such as phase shifters, reconfigurable filters, tuners and other planar circuits. In order to determine the potential of LCP as the substrate for MEMS switches, Clamped-clamped (bridge-type) and clamped-free (cantilever-type) coplanar waveguide (CPW) 
switches with a membrane size of $100 \square 200 \square \mathrm{m}^{2}$ and various hinge geometries (solid and meander shaped) have been fabricated on LCP substrates with a $3 \square \mathrm{m}$ thick polyimide layer in order to planarize the surface using a simple four mask low-temperature process. The CPW signal lines were fabricated by evaporating $\mathrm{Ti} / \mathrm{Au} / \mathrm{Ti}$ $(400 \AA / 5000 \AA / 400 \AA)$. Then PECVD $\mathrm{Si}_{3} \mathrm{~N}_{4}$ layer was patterned between the membrane and the signal line. A $1.8 \square \mathrm{m}$ thick photoresist (1813) was spin coated and patterned to create the air-gap. $\mathrm{Ti} / \mathrm{Au} / \mathrm{Ti} \quad(400 \AA / 3000$ $\AA / 300 \AA$ ) seed layer was then evaporated and patterned and electroplated. Finally, after removing the sacrificial photoresist layer with a resist stripper, a critical point drying process was used to release the switches.

Scanning Electron Microscope (SEM) picture of the fabricated air-bridge type CPW switch structure with a $1.2 \square \mathrm{m}$ thick gold membrane, a $1.8 \square \mathrm{m}$ air-gap and a membrane size of $100 \times 200 \mathrm{~mm}^{2}$, is shown in fig. 4 .

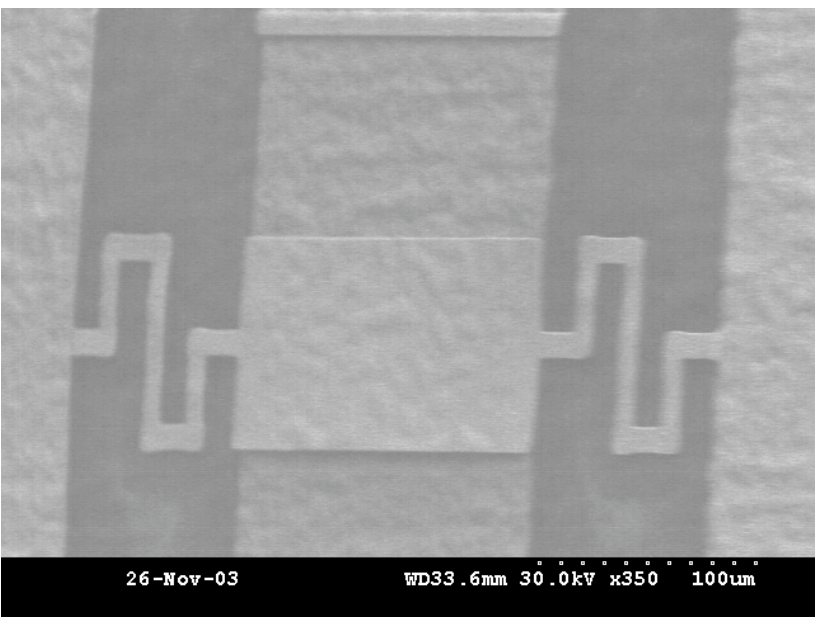

Fig 4. SEM of a fabricated airbridge type CPW switch on LCP with $1.2 \square \mathrm{m}$ thick Au membrane and meander-shaped support

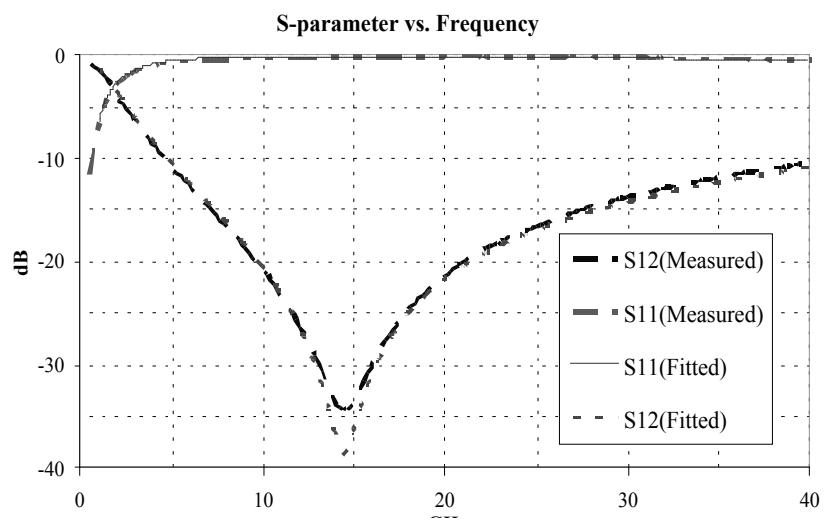

Fig 5. Measured and Simulated S-parameters for the Airbridge switch with $\mathrm{SiN}$ as dielectric in DOWN state
The measured results, shown in Figs. 5-6, were curvefitted in order to extract the switch model. The pull-in voltage was measured to be $20 \mathrm{~V}$. When the air-bridge switch is activated, the insertion loss is around $0.13 \mathrm{~dB}$ at $20 \mathrm{GHz}$ and $\mathrm{C}_{\mathrm{ON}}=3.5 \mathrm{pF}$, while the return loss is around $20 \mathrm{~dB}$ at $20 \mathrm{GHz}$. When the switch is in the UP position, $\mathrm{C}_{\mathrm{OFF}}=35 \mathrm{fF}$. From the results, the switch on LCP substrate has extremely low loss. It is flexible and is compatible with the required package. It enables integration of reconfigurable architectures on LCP.

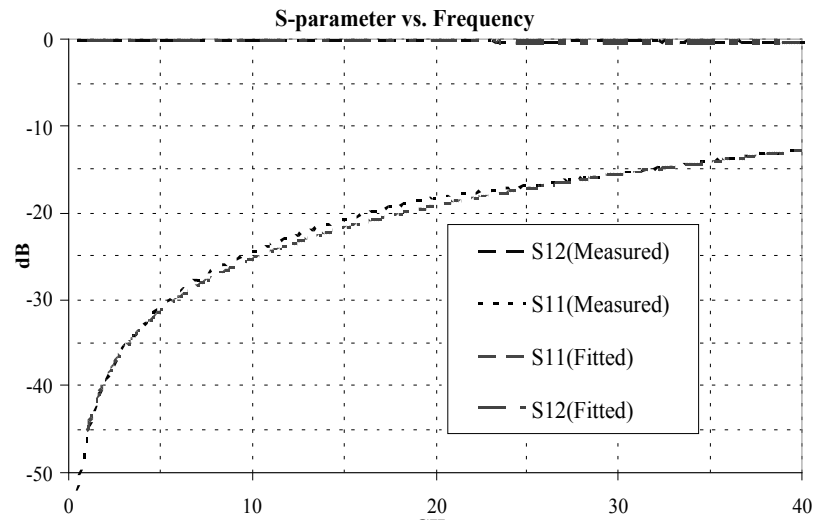

Fig 6. Measured and Simulated S-parameters for the Airbridge switch with $\mathrm{SiN}$ as dielectric in UP state

\section{WLAN MODULE IMPLEMENTATION}

In this section we demonstrate a functional RF module compliant with the IEEE 802.11a WLAN applications, incorporating LCP board technology. The architecture demonstrated is a super heterodyne $\mathrm{Tx} / \mathrm{Rx}$ system. Two passive mixers, achieving higher linearity, up-convert the low IF (20 MHz) OFDM signal to the 5.x GHz frequency band (fig 7) and two BPF operations cancel the unwanted images after each mixing.

Driver stages provide the gain needed to balance out the losses due to the passives, while the PA module demonstrating a $\mathrm{P}_{1 \mathrm{~dB}}$ of $30 \mathrm{dBm}$ enables the operation at a back off of $6 \mathrm{~dB}$, which is a prerequisite for OFDM transmission.

Inspection of the frequency spectrum (Fig.8) of the signal at the output of the Tx module shows that the leakage of the local oscillator signal, as well as the leakage of the unwanted image at $\mathrm{LO}_{2}-\mathrm{LO}_{1}$, are efficiently suppressed by $55 \mathrm{~dB}$ to the level of $-48 \mathrm{dBm}$.. The receiver shows overall $\mathrm{NF}$ of lower than $8 \mathrm{~dB}$ in order to enable for the proper RF reception and the demodulation of signals as low as $-70 \mathrm{dBm}$. 


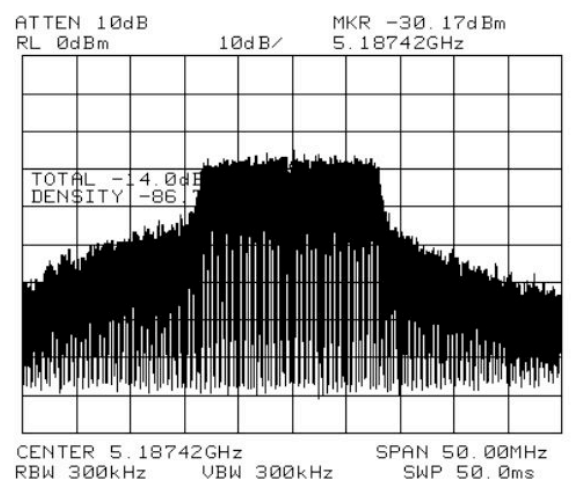

Fig 7. OFDM signal with carrier frequency $=5.18 \mathrm{GHz}$ and channel power $=-14 \mathrm{dBm}$

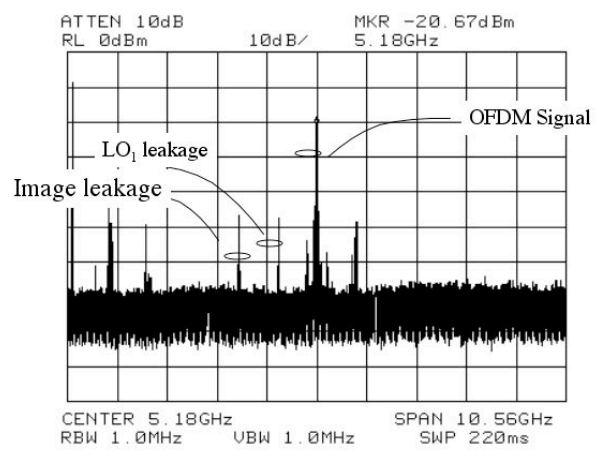

Fig 8. Image and $\mathrm{LO}_{1}$ cancellation in the receiver

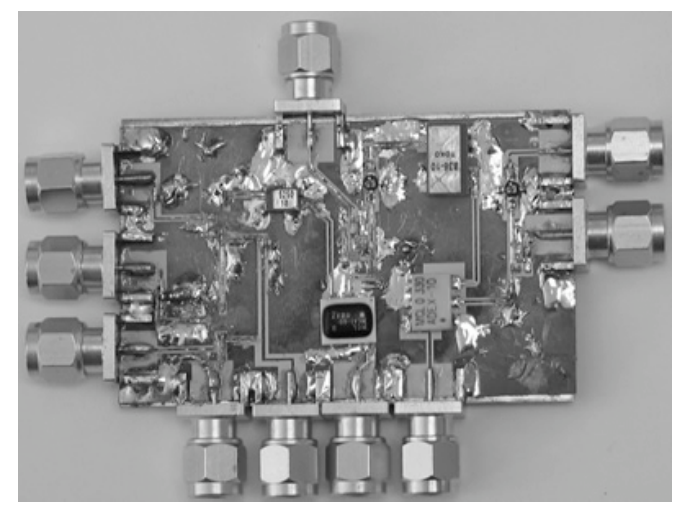

Fig 9. Photo of WLAN module

\section{CONCLUSION}

We have demonstrated the potential of LCP as the platform for multiband, reconfigurable integrated RF and mm-wave modules. SISO dual band filters with excellent loss performance for WLAN applications in $\mathrm{L}$ and $\mathrm{C}$ band (2.4dB in $\mathrm{L}$ band and $1.8 \mathrm{~dB}$ in $\mathrm{C}$ band respectively) have been reported. Extremely low loss $(0.1 \mathrm{~dB}$ up to $20 \mathrm{GHz})$ and flexible switches have been fabricated on LCP material. Finally the integration of WLAN module using LCP board technology shows great potential for miniaturized, low cost solutions for wireless communication systems. The receiver shows a high sensitivity $(\sim-70 \mathrm{dBm})$, low noise figure $(<8 \mathrm{~dB})$ and high LO leakage suppression of $55 \mathrm{~dB}$. The transmitter works at a $6 \mathrm{~dB}$ back off from output $\mathrm{P}_{1} \mathrm{~dB}$ of $30 \mathrm{dBm}$. As a conclusion, LCP constitutes an all-in-one solution for the heterogeneous SOP integration for multiband and reconfigurable RF and mm-wave applications.

\section{ACKNOWLEDGEMENT}

The authors wish to acknowledge the support of the NSF CAREER ECS-9984761, the NSF ECS-0313951, the NASA NCC3-1015, the Georgia Electronic Design Center and the Georgia Tech Packaging Research Center.

\section{REFERENCES}

[1] S. Pinel, M. Davis, V. Sundaram, K. Lim, J. Laskar, G. White and R. Tummala "High Q passives on Liquid Crystal Polymer substrates and $\square \mathrm{BGA}$ technology for 3D integrated RF Front-end Module" IEICE Trans. On Electronics, Aug 2003 vol. E86-C No. 8 Page: 15841592

[2] D. Thompson, P. Kirby, J. Papapolymerou, M. M. Tentzeris, "W-Band Characterization of Finite Ground Coplanar Transmission Lines on Liquid Crystal Polymer (LCP) substrates", Proc IEEE Electronic Components and Technology Conference, 2003 pp.1652-1655 New Orleans, LA, May 2003.

[3] M. F. Davis, S. W. Yoon, S. Pinel, K. Lim, J. Laskar, "Liquid Crystal Polymer-based Integrated Passive Development for RF Applications", Microwave Symposium Digest. 2003 IEEE MTT-S International, vol. 2 pp.1155-1158 Philadelphia, PA, June 2003.

[4] C. Quendo, E. Rius, C. Person, "Narrow bandpass filters using dual-behavior resonators," IEEE Trans. On Microwave Theory and Techniques, vol.51, n.3, Page: 734-743, March 2003.

[5] S. P. Pacheco, L. P. B. Katehi and T. C. Nguyen, "Design of Low Actuation Voltage RF MEMS Switch", Microwave Symposium Digest. 2000 IEEE MTT-S International, Page(s): 165 -168 vol.1. 